\section{The Central Governor Model in 2012: eight new papers deepen our understanding of the regulation of human exercise performance}

\author{
Timothy D Noakes
}

Why should a journal of sports and exercise medicine include an extensive series of papers that deal exclusively with exercise physiology; indeed with a tiny subsection of that discipline? The reason is perhaps twofold. The first is that the published articles all address the novel aspects of exercise-induced fatigue and the regulation of human exercise performance.

Fatigue remains a common complaint that brings patients to their primary care physicians. Surely there must be some common explanation for the types of fatigue that we feel, whether at rest or during exercise? Can the exercise sciences contribute something unique to our understanding of this important clinical phenomenon?

In 1923, Nobel Laureate Archibald V Hill developed the currently popular model of exercise fatigue. ${ }^{1}$ According to his understanding, fatigue develops in the exercising skeletal muscles when the heart is no longer able to produce a cardiac output which is sufficient to cover the exercising muscles' increased demands for oxygen. This causes skeletal muscle anaerobiosis (lack of oxygen) leading to lactic acidosis. The lactic acid so produced then 'poisons' the muscles, impairing their function and causing all the symptoms we recognise as 'fatigue'. The collection of articles in this issue provides several invigorating challenges to Hill's hallowed interpretation. Which brings us to the second reason why these articles are published here rather than elsewhere. For some of the articles included were reviewed unfavourably elsewhere; perhaps they were considered heretical. Clearly, some believe that ideas challenging a hallowed dogma are best left unpublished. Otherwise, we might need to update our thinking. Or worse, our teaching!

Correspondence to Timothy D Noakes, Department of Human Biology, University of Cape Town, Research Unit for Exercise Science and Sports Medicine, Newlands

7725, South Africa; noakes@iafrica.com

\section{WHEN IS V0 2 REALLY 'MAX'?}

The issue begins with the complementary studies of Lex Mauger et al from the University of Bedfordshire and Fernando Beltrami and his associates ${ }^{3}$ from the University of Cape Town. Their studies disprove perhaps the most fundamental idea on which Hill based his entire philosophy. According to Hill, during the period between progressive exercise and exhaustion, whole body oxygen consumption reached a maximum value - the maximum oxygen consumption $\left(\mathrm{VO}_{2 \max }\right)$ - 'beyond which no effort can drive it'. Independently, both research groups show that the $\mathrm{VO}_{2 \max }$ measured as Hill described is not the absolutely highest value of which the athlete is capable. Had Hill shown this in 1923, he could not have concluded that maximal exercise performance is controlled by a limiting cardiac output. Instead a more complex explanation is required to explain why athletes always terminate exercise before they reach an ultimate oxygen limitation.

If failure of oxygen delivery was to limit exercise performance, then it follows, that those with the highest $\mathrm{VO}_{2 \max }$ values must be the most successful. This would mean that we could do away with Olympic competitions in endurance events. Instead medals could simply be distributed more easily to those athletes with the highest values. But it has been known since at least the early 1960s that the best athletes do not always have the highest $\mathrm{VO}_{2 \max }$ values.

Lamberts et $a l^{4}$ wondered if there is additional information collected during the $\mathrm{VO}_{2 \max }$ test that might aid in the prediction of athletic ability. They show that, when scaled appropriately to body mass, the peak power output achieved during a maximal cycling test predicts $40 \mathrm{~km}$ time trial performance with remarkable accuracy. Since this form of testing requires little sophisticated equipment and is less demanding than real competition, they propose that it may be the ideal method for monitoring a cyclist's state of physical preparedness and the response to training.

Another prediction of the Hill model is that the measurement of blood lactate concentrations during exercise can be used to define both the precise exercise training intensities that will produce the greatest training benefits as well as the extent to which those training adaptations have occurred. Using careful statistical analysis, Morton et a $\left.\right|^{5}$ from Massey University, New Zealand, establish that the individual variability in the blood lactate response to exercise is too large to allow such firm conclusions. Rather, they conclude that unrealistically large changes in power output would have to occur before it can be claimed with certainty that training has produced a real change in an individual's blood lactate concentrations during exercise. These findings should encourage sober reflection among that large group of exercise scientists who use blood lactate concentrations to guide athletes' training.

As long as lactic acid was considered to be the sole 'exercise stopper', it was natural to presume that this molecule is also the regulator of the pace that athletes chose during exercise. But this interpretation was discounted when it was realised that a single molecule cannot explain why athletes start exercise at different paces depending on the expected exercise duration, nor why they speed up near the end of exercise. These common observations can be explained only by the presence of specific neural control mechanisms. ${ }^{6}$ But since the Hill model promotes a concept of 'brainless' physiology, it provides no scope for any role for the brain in the regulation of exercise performance.

\section{PACING INSIGHTS - A KEY TO RACING}

To determine whether information provided to untrained subjects during exercise can influence their chosen pacing strategies, perhaps through conscious brain mechanisms, Professor Williams et al from the University of Exeter compared pacing strategies during successive $4 \mathrm{~km}$ cycling time trials in the laboratory. During the trials, subjects received either no information about how far they had travelled or they received complete information. They showed that this knowledge was without effect in untrained subjects whereas this information can improve the performances of experienced cyclists. Thus, they concluded that task familiarity and training status influence the ability to learn a pacing strategy and that 
this requires time to train the appropriate brain mechanisms. Indeed it is known that proper pacing is perhaps the most difficult skill for neophyte athletes to learn.

Dr Carl Foster's group at the University of Wisconsin-LaCrosse in collaboration with the group of J J De Koning at the Vrye University, Amsterdam have been at the forefront of studying the biology of athletic pacing particularly in exercise of quite short duration (min). By comparing the best and worst $1500 \mathrm{~m}$ cycling time trial performances of a group of trained cyclists, they show that from the very start of exercise, experienced athletes adopt an optimum pacing strategy but which differs from test to test. ${ }^{9}$ Thus athletes begin their best performances at a faster pace and sustain a higher power output throughout the effort than when they perform less well. The above study concluded that experienced athletes are able to adjust their performance on a day-to-day basis via physiological and perhaps other cues interpreted even at the very start of the exercise bout. They speculate that specific brain areas could be involved: the dorsal posterior insula collects afferent sensory information about the homeostatic state of the body tissues whereas the right anterior insula generates a 'feeling' or sensation based on that information. They propose that the pacing strategy 'seems to be an internal negotiation from the start onwards, comparing actual sensed fatigue with the expected state of fatigue, where the rate of perceived exertion (RPE) might be thought of as the conscious interpretation of the actual fatigue'.

Pires et al from the University of Sao Paulo and John Hammond from the University of Canberra used a novel deception to determine the extent to which the sensations generated during exercise, specifically the RPE, regulates the pacing strategy. ${ }^{10}$ They instructed the athletes to exercise until exhaustion at the same absolute work rate on two separate occasions. However, subjects in one of those exercise bouts were deceptively informed that they would be exercising at a substantially lower work rate that would produce a much lower RPE. However, this deception did not work. Instead, subjects correctly perceived both exercise bouts to be equally stressful and reported identical RPE values during both bouts. The above study concluded that the subjects were able to accurately determine the intensity of the effort and that the RPE appears to be a regulator of the exercise performance since the deception did not alter the relationship between the RPE and the exercise duration.

\section{DISTINGUISHING PHYSICAL SENSATION} FROM NEURAL EFFORT (TEA SCALE)

The paper by Swart et al from the University of Cape Town ${ }^{11}$ represents another significant advance in our understanding of the nature of the symptoms of fatigue that develop during exercise and the manner in which they interact to determine the exercise performance. These authors wished to determine whether the traditionally described RPE scale measures both the physical sensations produced by exercise and the physiological/psychic effort required to perform the task. They note that in his original description Borg described the RPE as a measure of an 'individual's total physical and psychic reaction to exertion'. ${ }^{12}$ Thus they set out to separate the physical sensations produced by the actual performance of the work from those psychic or psychological sensations which represent the neural effort of maintaining a given level of physical work. This later group of sensations - the sense of effort - are loosely defined as subjective sensations not based on any known physiological changes induced by exercise but which are generated by the brain in response to as yet unidentified specific components of the exercise bout. They further postulated that the sense of effort would serve a biological purpose - in particular the maintenance of homeostasis - so that it would rise only when the exercise was of such an intensity or duration that it threatened the homeostasis. A rising sense of effort would then force the subject to reduce the exercise intensity in order to prevent a catastrophic biological failure.

To distinguish changes in the physical symptoms produced by exercise from those measuring the sense of effort, they studied subjects who had been carefully instructed on how to use the Borg RPE scale to measure only the physical symptoms they experienced during exercise. To quantify their sense of effort - the effort of maintaining the work rate - they were instructed the use of a novel scale - the Task Effort and Awareness (TEA) scale.

Subjects then completed two $100-\mathrm{km}$ cycling bouts, one at a maximal and the other at a submaximal effort. A series of all-out 1-km sprints were included in both exercise bouts. The key was that subjects were instructed to perform all these sprints with an absolutely maximal effort.

The findings showed that whereas RPE rose progressively during exercise in both trials and was lower in the submaximal trial, it reached a maximal value of 19 only in the final sprint in both trials. In contrast, the TEA score was maximal at the end of each sprint even during the submaximal trial in which each sprint began at a lower TEA (and RPE) score.

Thus, their novel finding is that the TEA score measures something other than the physical sensations induced by exercise and which are adequately captured by the RPE. They conclude that the TEA score measures the psychic effort of sustaining the physical effort and is directed by the brain centres that regulate homeostatic afferent feedback in multiple organs in relation to how far the exercise still has to go. Therefore 'the conscious decision of whether to maintain, increase or decrease the current workload or indeed to terminate the exercise altogether may be the outcome of a balance between motivation and affect and the sensation that is defined as the sense of effort'.

\section{PRACTICAL IMPLICATIONS}

This important collection of studies significantly advances our understanding of the factors that regulate exercise performance. They provide strong evidence that Hill over-interpreted the finding from his original relatively simple experiments. The studies of Mauger and Sculthorpe ${ }^{2}$ and Beltrami et a $\beta$ should insure that no longer can Hill's classic experiments be used as absolute proof that a maximal cardiac output 'limits' maximal exercise performance as a result of a 'poisonous' lactic acidosis.

Instead, the detailed studies of pacing reported here show that complex brain mechanisms are able to determine the physical state of the body on a momentto-moment basis and to adjust the work output specifically to insure that exercise can be conducted safely without the development of a catastrophic biological failure. The study of Swart et all1 shows that the brain uses two conscious sensations to regulate that exercise - the physical symptoms produced by the exercise and measured by the RPE and the sense of effort, which is a gauge of the psychic effort required to sustain a given work output. Thus 'The direct consequence of the increasing sense of effort will be an altered behaviour, specifically a voluntary reduction in the exercise intensity. Conversely, exercise intensities that do not pose a threat to homeostatic control produce no or little sense of effort'.

Were he alive today, I suspect that the person most eager to embrace our new wisdom would have been Hill himself. He would have appreciated that the refutation of his ideas that have done so much to define the modern exercise sciences can only be of the greatest value to the 
future growth of the discipline he helped to establish.

\section{Competing interests None.}

Provenance and peer review Commissioned; internally peer reviewed.

Received 25 November 2011

Accepted 25 November 2011

Br J Sports Med 2012;46:1-3. doi:10.1136/bjsports-2011-090811

\section{REFERENCES}

1. Noakes TD. How did A V Hill understand the VO and the "plateau phenomenon"? Still no clarity? Br J Sports Med 2008;42:574-80.

2. Mauger AR, Sculthorpe N. A new $\mathrm{VO}_{2 \max }$ protocol allowing self-pacing in maximal incremental exercise. Br J Sports Med 2011:46:59-63.
3. Beltrami FG, Froyd C, Mauger A, et al.

Conventional testing methods produce sub-maximal values of maximum oxygen consumption. $\mathrm{Br} \mathrm{J}$

Sports Med 2012:46:23-9.

4. Lamberts RP, Lambert Ml, Swart J, et al. Allometric scaling of peak power output accurately predicts time trial performance and maximal oxygen consumption in trained cyclists. Br J Sports Med 2011:46:36-41.

5. Morton RH, Stannard SR, Kay B. Low reproducibility of many lactate markers during incremental cycle exercise. Br J Sports Med 2011;46:64-9.

6. Noakes TD. The central governor model of exercise regulation applied to the marathon. Sports Med 2007;37:374-7.

7. Noakes TD. Testing for maximum oxygen consumption has produced a brainless model of human exercise performance. Br J Sports Med 2008;42:551-5.

8. Williams CA, Bailey SD, Mauger AR. External exercise information provides no immediate additional performance benefit to untrained individuals in time trial cycling. Br J Sports Med 2011;46:49-53.

9. Hettinga FJ, De Koning JJ, Schmidt LJ, et al. Optimal pacing strategy: from theoretical modelling to reality in 1500-m speed skating. Br J Sports Med 2011;45:30-5.

10. Pires FO, Noakes TD, Lima-Silva AE, et al. Cardiopulmonary, blood metabolite and rating of perceived exertion responses to constant exercises performed at different intensities until exhaustion. Br J Sports Med 2011:45:1119-25.

11. Swart J, Lindsay TR, Lambert Ml, et al. Perceptual cues in the regulation of exercise performance physical sensations of exercise and awareness of effort interact as separate cues. Br J Sports Med 2011;46:42-8.

12. Borg G. A simple rating scale for use in physical work tests. Kungliga Fysiografi ska Sallskapets I Lund Forhandlinger 1962;32:7-15. 